\title{
Efficacy of Itraconazole Versus Terbinafine for the Treatment of Tineacruris.
}

Nabin Bhakta Shakya ${ }^{1}$, Sagar Mani Jha ${ }^{1}$, Anil Dangol', Sunil Shakya ${ }^{1}$, Aparna shah $^{1}$.

${ }^{1}$ Department of Dermatology and Venereology, Shree Birendra Hospital

\begin{abstract}
Introduction: Tenia Cruris is the commonest dermatophyte infection which is common in men and aggravated by warm humid condition Teniarubrum is the main species for the disease ${ }^{1,2}$. Itraconazole and Terbinafine are the better newer drugs for the therapeutic option due to their efficacy, convenient and less side effect. Itraconazole is a wider spectrum Thiazole group of fungistatic drugs which inhibit sterol $14 \alpha$ demethylase impairing biosynthesis of fungal ergosterol. Similarly Terbinafine is broad specturmallylamine group of fungicidal activity which inhibits squalene 2, 3 epoxidase enzyme thus inhibit fungal biosynsthesis of ergostero ${ }^{3-5}$. These two drugs have been needed conventionally as a pulse and continuous therapy. The main aim of this study is to compare the efficacy of both drugs and to recommend the effective treatment in case of Teniacruris.
\end{abstract}

Methods: In this randomized comparative study, the efficacy and safety of oral itraconazole $(n=35)$ and terbinafine $(n=35)$, each given for 2 weeks, was compared in patients with tineacruris within the period of 1 year study.

Results: The final evaluation at week 4 showed a cure rate of $91.4 \%$ for itraconazole and $82.9 \%$ for terbinafine. No definite adverse effect was noted.

Conclusions: Itraconazolehas high cure rate and less failure rate without side effects in comparision toTerbinafine.

Keywords: itraconazole, teniacruris, terbinafine

\section{INTRODUCTION}

Among the dermatophytes, TeniaCruris infection is common in groin region. Men suffer more than female and it is frequent in warm humid tropical condition. Itching erythematous plaque with scales are the typical clinical feature ${ }^{1,2}$.

The disease can be controlled by many options. There are so many studies regarding the treatment of Tenia infection. Among them Itraconazole and Terbinafine are the better newer drugs for the therapeutic option due to their efficacy, convenient and less side effect. Itraconazole is a wider spectrum Thiazole group of fungistatic drugs which inhibit sterol $14 \alpha$ demethylase impairing biosynthesis of fungal ergosterol. This drug is metabolized in liver with extensive tissue binding. May have GIT distress at higher doses and increase serum aminotransferase ${ }^{3}$. Similarly Terbinafine is broad specturmallylamine groups of fungicidal activity which inhibit squalene 2, 3 epoxidase enzyme thus inhibit fungal biosynsthesis of ergosterol. This drug is lipophilic, widely distributed in the body and strongly plasma protein bound. Common side effect may be GIT upset and rashes ${ }^{4,5}$. These two drugs have been needed conventionally as a pulse and continuous therapy in case of any sorts of dermatophyte infections.

\section{Correspondence:}

\section{Lt. Col. Dr. Nabin Bhakta Shakya}

Department of Dermatology and Venereology, Shree Birendra Hospital

Kathmandu, Nepal

Email: docnabin@yahoo.com

Phone: +977-9843012310 
The main aim of this study is to compare the efficacy of both drugs and to recommend the effective treatment in case of Teniacruris.

\section{METHODS}

A total of eighty patients including both male and female of age between 18 and 40 years, suffering from mycologically confirmed tineacruris by $10 \%$ Potassium Hydroxide preparation within the period of one year were included in this study. Patients were randomly allocated either to itraconazoleorterbinafine group. Patients were excluded if they had allergy to drugs, used oral antifungals within 8weeks or topical antifungals within 4weeks before screening, concurrent therapy with rifampicin, phenytoin, digoxin, oral anticoagulants, cyclosporin, astemizole and terfenadine, psoriasis of the scalp, history of any systemic illness or abnormal liver and renal function tests. After taking written consent from the enrolled patients, they were randomlygiven either itraconazole or terbinafinefrom the outpatient department. Both drugs were given orally for a period of 2 weeks. The itraconazole was given 100 $\mathrm{mg}$ twice daily whereas $250 \mathrm{mg}$ ofterbinafinewas given once daily. During the screening visit, a detailed medical history was obtained, and a thorough examination was performed. Various clinical signs and symptoms were rated according to a four-point scale from 0 to 3 ( 0 =absent, $1=$ mild, $2=$ moderate and $3=$ severe). After commencement of therapy, patients were followed up at weeks 2 (end of treatment period) and 4. At each visit, a clinical assessment was made, and material was taken for mycological examination. The therapeutic efficacy was evaluated at 4 weeks. Mycological samples (skin scrapings) were subjected to $10 \%$ potassium hydroxide wet mounts. Blood was tested for biochemical and haematological profile at baseline and then repeated at the end of weeks 4. The assessment of efficacy was based on the combined evaluation of mycological results and the sum of clinical scores at week 4 according to the following scheme.

\section{Effective therapy included:}

a. Complete cure: non residual clinical signs and symptoms except post inflammatory hyperpigmentation with negative microscopic findings.

b. Mycological cure: minimal residual signs andsymptoms (sum score<2) with negative mycology.

\section{Ineffective therapy comprised:}

a. Improvement: positive mycology in microscopy with significant (>50\%) clinical improvement or negative mycology with no clinical improvement.

b. Failure: non clinical or mycological improvement ${ }^{2}$.
Adverse events were recorded on physical examination or laboratory investigations. Chi-square test was used for statistical analysis.

\section{RESULTS}

Out of 80 patients enrolled, only 70 were evaluated. 10 patients were excluded because of irregular follow up. Thirty five patients were treated with itraconazole and 35 patients with terbinafine. The mean age was 29.3 years and male female were equally evolved as in table 1 . At the end of week 4, positive mycology had fallen from $100 \%$ to $8.6 \%$ and $17.1 \%$ in itraconazole and terbinafine groups respectively. Trichophytonrubrumwas the predominant pathogen in itraconazole $(n=27)$ and terbinafine $(n=28)$ groups. Epidermophytumfloccosum was found in 8 in itraconazole and 7 in terbinafine groups respectively. After 4 weeks, $91.4 \%$ and $82.9 \%$ patients were considered to be effective therapy in the itraconazole and terbinafine groups, respectively, as shown in Table 2 . All the failures in both groups [itraconazole $(n=1)$ and terbinafine $(n=4)$ ] had tineacruris. None of the patients showed any significant side effect in both Itraconazole and Terbinafine groups.

Table 1. Characteristic of Patients in either group

\begin{tabular}{|c|c|c|c|}
\hline \multirow[t]{2}{*}{ Characteristic } & \multirow{2}{*}{$\begin{array}{c}\text { Number } \\
(\%)\end{array}$} & \multicolumn{2}{|c|}{ Group } \\
\hline & & Itraconazole & Tebinafine \\
\hline Patients & 70 & 35 & 35 \\
\hline $\begin{array}{l}\text { Sex distribution: } \\
\text { Male } \\
\text { Female }\end{array}$ & $\begin{array}{l}60(85.7) \\
10(14.3)\end{array}$ & $\begin{array}{c}30 \\
5\end{array}$ & $\begin{array}{c}30 \\
5\end{array}$ \\
\hline $\begin{array}{l}\text { Mean } \\
\text { Range }\end{array}$ & $\begin{array}{c}29.3 \text { yrs. } \\
18-40\end{array}$ & $\begin{array}{c}29.4 \text { yrs. } \\
18-40\end{array}$ & $\begin{array}{c}29.2 \text { yrs. } \\
18-40\end{array}$ \\
\hline $\begin{array}{l}\text { Age distribution: } \\
\qquad \begin{array}{l}\leq 20 \\
21-25 \\
26-30 \\
31-35 \\
36-40\end{array}\end{array}$ & $\begin{array}{c}3(4.3) \\
20(28.6) \\
20(28.6) \\
12(17.1) \\
15(21.4)\end{array}$ & $\begin{array}{c}1 \\
10 \\
10 \\
6 \\
8\end{array}$ & $\begin{array}{c}2 \\
10 \\
10 \\
6 \\
7\end{array}$ \\
\hline
\end{tabular}

Table 2. Comparative results of two drugs at 4 weeks

\begin{tabular}{|l|l|c|c|c|}
\hline \multirow{2}{*}{ Effectiveness } & \multicolumn{1}{|c|}{ Results } & \multicolumn{2}{|c|}{ Drug } & \multirow{2}{*}{ Total } \\
\cline { 3 - 5 } & & Itraconazole & Tebinafine & Totfective \\
therapy & Complete cure & 28 & 25 & 53 \\
\cline { 2 - 5 } & $\begin{array}{l}\text { Mycological } \\
\text { cure }\end{array}$ & 4 & 4 & 8 \\
\hline $\begin{array}{l}\text { Ineffective } \\
\text { therapy }\end{array}$ & Improvement & 2 & 2 & 4 \\
\cline { 2 - 5 } & Failure & 1 & 4 & 5 \\
\hline
\end{tabular}


Fig 1. Effects of Itraconazole Fig 2. Effects of Terbinafine
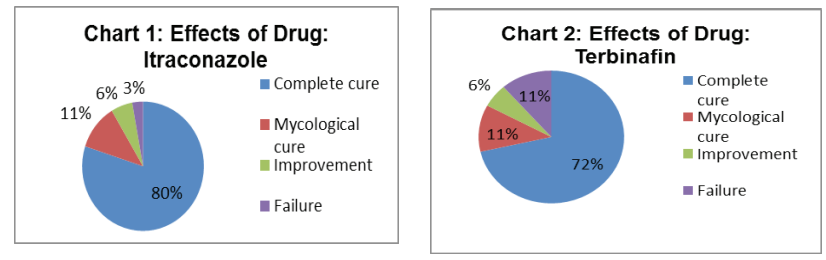

\section{DISCUSSION}

Any new therapy for tineacruris should bring about rapid clinical and mycological cure without relapse and possess fewer side-effects. The results of our study suggest that both these drugs fulfill the above criteria equally well. Subjective and objective clinical improvement, negative mycology and efficacy at week 4 do not show any significant difference. These results demonstrate that both drugs are highly effective when given for 2 weeks. As T. rubrum was the major pathogen, it appears that 2 weeks of therapy with either itraconazole or terbinafine is effective in eradicating this infection in most of the patients. However, all 9 non-responders had this dermatophyte, suggesting that the duration of therapy has to be extended beyond 2 weeks in certain cases of T. rubrum infection. Although the number of cases resulting from other pathogens was small, they do respond to this short-term therapy with drug usually in Tenia capitis. ${ }^{6-13}$ Bothitraconazole and terbinafine are highly lipophilic and keratinophilic. They persist in the stratum corneum and hair in high concentrations for 3-4 weeks after therapy is discontinued. These levels are well above the minimum inhibitory concentrations for most of dermatophytes, and hence sufficient to inhibit fungal growth ${ }^{14}$. This enables both these drugs to be effective as short-term therapy for tineacruris.Although Terbinafine is more compliance for the patients due to only once daily dose and less cost effective than Itraconazole. In our study, both drugs are safe and well tolerated.

\section{CONCLUSIONS}

Itraconazolehas high cure rate and less failure rate without side effects in comparision to Terbinafine. Although Terbinafineis better in terms of compliance and cost the failure rate is relatively higher.

\section{REFERENCES}

1. Wolff K, Lowell A, Barbarra A, David J. Fitzpatrick's Dermatology in general medicine, seventh edition. 2003;2:pp1815.

2. Hay RJ, Ashbee HR. Rook's Textbook of Dermatology, Eighth Edition 2010;pp1-93.
3. Hans CK, Claudia S. The significance of itraconazole for treatment of fungal infections of skin, nails and mucous membranes. Journal of The German Society of Dermatology.2009;7:11-9.

4. Gupta AK, Adamiak A, cooper EA. The Efficacy and Safety of Terbinafine in Children. Journal of the European Academy of Dermatology and Venereology. 2003;17:627-40.

5. Chapel KL, Rasmussen JE. Pediatric dermatology: advances in therapy. J Am AcadDermatol. 1997;36:513-26.

6. Lopez-Gomez S, Del Palacio A, van Cutsem J et al. Itraconazole versus griseofulvin in the treatment of tineacapitis: a double-blind randomized study in children. Int J Dermatol.1994;33:743-7.

7. Lukacs A, Korting HC, Linder A. Successful treatment of griseofulvin-resistant tineacapitis in infants. Mycoses. 1994; 37:451-63.

8. Elewski B. Tineacapitis: itraconazole in Trichophytontonsurans infection. J Am AcadDermatol. 1994;31:65-7.

9. Gupta $\mathrm{AK}$, Alexis ME, Raboobee $\mathrm{N}$ et al. Itraconazole pulse therapy in treatment of tineacapitis in children: an open multicenter study. $\mathrm{Br} J$ Dermatol.1997;137:251-4.

10. Haroon TS, Hussain I, Mahmood A et al. An open clinical pilotstudy of the efficacy and safety of oral terbinafine in dry non-short Course Therapy in TeniaCapitis. British Association of Dermatologists. British Journal of Dermatology. 1998;139:672-4.

11. Nejjam F, Zagula M, Cabiac MD et al. Pilot study of terbinafine in children suffering from tineacapitis: evaluation of efficacy, safety and pharmacokinetics. Br J Dermatol. 1995;132:98-105.

12. Haroon TS, Hussain I, Aman S etal.A randomized, double-blind comparative study of terbinafine vs. griseofulvin in tineacapitis. JDermatol Treat.1995;6:167-9.

13. 13 Haroon TS, Hussain I, Aman S etal.A randomized double-blind comparative study of terbinafine for 1, 2 and 4 weeks in tineacapitis. $\mathrm{Br} \mathrm{J}$ Dermatol.1996;135:86-8.

14. 14 Kullavanijaya P, Reangchainam S, Ungpakorn R. Randomized single-blind study of efficacy and tolerability of terbinafine in the treatment of tineacapitis.J Am AcadDermatol. 1997;37:272-3. 